\title{
Orthodontics in the era of big data analytics
}

\author{
Veerasathpurush Allareddy ${ }^{1}$ (D) | Shankar Rengasamy Venugopalan ${ }^{2}$ | Romesh P. Nalliah ${ }^{3}$ | \\ Jennifer L. Caplin ${ }^{1}$ | Min Kyeong Lee ${ }^{1}$ | Veeratrishul Allareddy ${ }^{4}$
}

\author{
${ }^{1}$ Department of Orthodontics, University \\ of Illinois at Chicago College of Dentistry, \\ Chicago, Illinois \\ ${ }^{2}$ Department of Orthodontics and \\ Dentofacial Orthopedics, University of \\ Missouri at Kansas City School of Dentistry, \\ Kansas City, Missouri \\ ${ }^{3}$ University of Michigan College of Dentistry, \\ Ann Arbor, Michigan \\ ${ }^{4}$ University of lowa College of Dentistry and \\ Dental Clinics, lowa City, lowa
}

\section{Correspondence}

Veerasathpurush Allareddy, Department of Orthodontics, University of Illinois at Chicago College of Dentistry, Chicago, IL. Email: sath@uic.edu

Shankar Rengasamy Venugopalan,

Department of Orthodontics and

Dentofacial Orthopedics, University of

Missouri - Kansas City School of Dentistry,

Kansas City, MO.

Email: rengavs@umkc.edu

Funding information

American Association of Orthodontists

Foundation Biomedical Research

\section{Structured Abstract}

The objective of this report was to provide an overview of the current landscape of big data analytics in the healthcare sector, introduce various approaches of machine learning and discuss potential implications in the field of orthodontics. With the increasing availability of data from various sources, the traditional analytical methods may not be conducive anymore for examining clinical outcomes. Machine-learning approaches, which are algorithms trained to identify patterns in large data sets, are ideally suited to facilitate data-driven decision making. The field of orthodontics is particularly ripe for embracing the big data analytics platform to improve decision making in clinical practice. The availability of omics data, state-of-the-art imaging and potential for establishing large clinical data repositories have favourably positioned the specialty of orthodontics to deliver personalized and precision orthodontic care. Specifically, we discuss about next-generation sequencing, radiomics in the context of $\mathrm{CBCT}$ imaging, and how centralized data repositories can enable real-time data pooling from multiple sources.

\section{KEYWORDS}

artificial intelligence, big data, machine learning, orthodontics research

\section{1 | THE CURRENT LANDSCAPE OF BIG DATA ANALYTICS}

Big data is almost always a reference to large data sets and/or the combination of all available data points drawn from multiple sources in order to recognize patterns that inform a customized experience for each individual. The concept of big data analytics has gained much prominence in the healthcare sector over the past few years. ${ }^{1,2}$ This trend can be attributed to availability of more data sources, fast processing computing resources and increasing focus on quality of care and clinical outcomes. A search in the PubMed database using the term "big data" indicated that total of 12643 articles were published in peer-reviewed journals as of 10/09/2018 (Figure 1). Of these, a vast majority (68\%) was published from 2013

Veerasathpurush Allareddy and Shankar Rengasamy Venugopalan these authors contrib uted equally. onwards. The definition of big data has evolved over the years and is largely reflective of the changes in data sources, data type, computing speed, storage capacity, data complexity and dimensionality. ${ }^{2}$ According to the National Institute of Health (NIH), data science is an "interdisciplinary field of enquiry in which quantitative and analytical approaches, processes, and systems are developed and used to extract knowledge and insights from increasingly large and/or complex sets of data." ${ }^{3}$ The terms big science, big data and big data analytics have been used interchangeably. It has come to be accepted that big data should comprise six fundamental qualities: volume, veracity, variety, variability, velocity and value. ${ }^{4,5}$ The advent of multiple data sources in the healthcare field such as electronic health records, omics data, imaging, biometrics, environmental data and patient collected information has armed us with an empirical datadriven framework to make clinical decisions. ${ }^{6}$ Yet, not all healthcare facilities and providers are equipped with the necessary resources in terms of trained personnel, core computing or the fundamental 


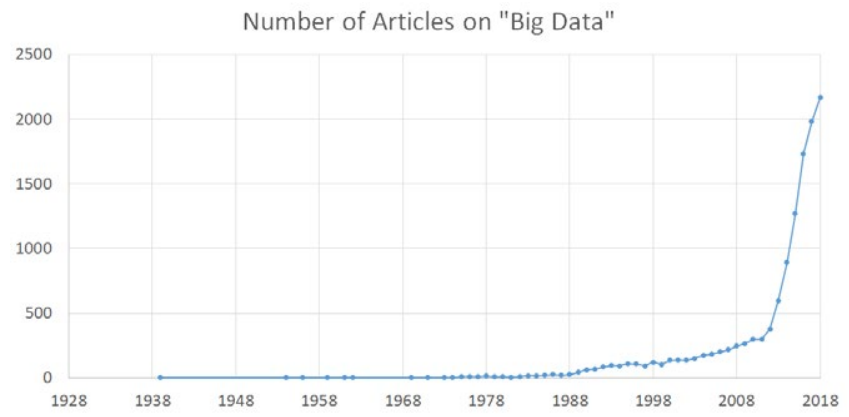

FIGURE 1 Number of articles on "Big Data" indexed in PubMed

knowledge to leverage the advantages of the big data platform. Specialties such as oncology, cardiology, surgery and radiology have been at the forefront of adapting to the big data environment and have made massive strides in using some of the big data analytical methods and data sources to deliver personalized and precision care. ${ }^{2,4-9}$ Using different data sources and new age big data analytic tools, these specialties have challenged the long-held traditional monolithic view of disease. ${ }^{4-12}$ Big data analytic tools have been successful in identifying personalized disease concepts and subtypes and delivering targeted aetiology-based therapies. ${ }^{10-12}$ This is reflected in the large number of peer-reviewed publications stemming from these fields documenting the individualized nature of care delivered. ${ }^{4-12}$

\section{2 | OVERVIEW OF BIG DATA ANALYTIC TOOLS}

Traditionally, clinical research has been centred on a model where there is a clearly defined hypothesis, primary outcomes, set of predictor variables selected on biological plausibility and experimental settings that are well controlled. The statistical methods that are used to test the study hypotheses, for example logistic regression for binomial outcomes and linear regression for continuous outcomes, work best under strict assumptions including lack of multicollinearity between variables, absence of endogeneity and model fitness. ${ }^{9,10,13-15}$ However, these assumptions do not hold when applied to real-world data. Patient-related variables tend to be related to each other to varying degrees, and it is almost impossible to lack endogeneity. Furthermore, with increasing availability of data from a multitude of sources and need for accounting for several layers of interactions between variables, the traditional methods may not be the best set of analytical tools to examine clinical outcomes in the big data environment.

In recent years, one of the big data analytic tools that has gained popularity in the applied health sciences is machine-learning algorithms. ${ }^{16}$ When the PubMed search engine was queried using the term "machine learning," 24132 articles were published as of 10/09/2018 (Figure 2). The number of articles on machine learning increased substantially since 2010 . The machine-learning approach, in contrast to traditional statistical approaches, is not based on a

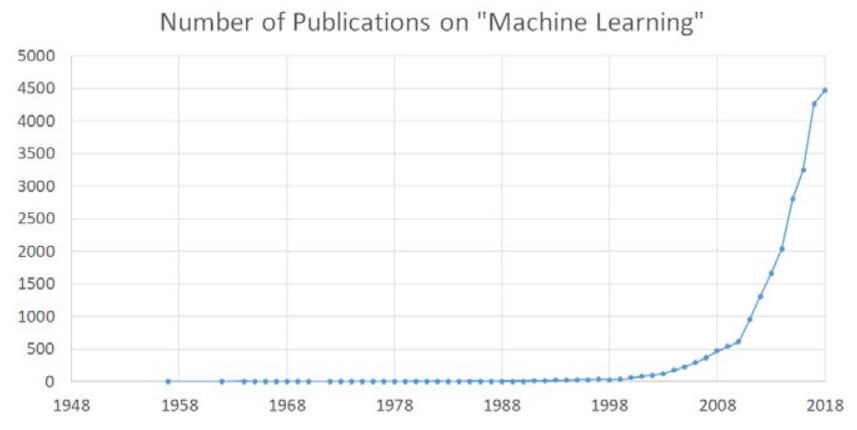

FIGURE 2 Number of articles on "Machine Learning" indexed in PubMed

study hypothesis nor is it dependent upon strict data parameters and data assumptions. ${ }^{10,11,14,16-18}$ Instead, the machine-learning approach examines patterns in data and can simultaneously account for multiple variables and interactions between variables and assumes that endogeneity is ubiquitous. ${ }^{10,11,14,16-18}$ Consequently, machine learning is the analytical approach of choice when we are inundated with big data drawn from multiple sources and in a wide range of formats. Machine learning has become increasingly utilized in health care in recent years and has been successful in identifying disease subtypes as well as providing insights into rare diseases and associated outcomes, predictive modelling, identification of never events and targeted therapies. ${ }^{10,11,14,16-18}$ Broadly, machine learning can be either supervised or unsupervised. In a supervised machine-learning approach, the algorithms are developed using a training data set and then are used to predict outcomes in a novel or real-world data set. ${ }^{10,11,14,16-18}$ Depending on the nature of the data and outcomes to be examined (eg mix of number of subjects versus number of features, linear or non-linear outcomes and dimensionality), various supervised machine-learning approaches are in vogue. The common methods include regularized regression, decision trees and support vectors. In an unsupervised machine-learning approach, the algorithms are programmed to identify patterns within a data set. Depending on hierarchical representation of data, clustering and dimensionality of data, frequently used approaches include neural networks, deep learning, tensor factorization and topological data analysis. ${ }^{10,11,14,16-18}$

\section{3 | IMPLICATIONS FOR ORTHODONTICS}

The last few decades have witnessed tremendous changes in our specialty. An increase in the number of patients with complex multidisciplinary problems seeking orthodontic care, the advent of temporary anchorage devices, a transition to a complete digital realm, newer imaging modalities and the availability of omics data have lent an entirely new dimension to the delivery of orthodontic care. We can no longer work in silos. A team-based approach to care transcending specialties that makes use of every data source is the new mantra. Some of the big data tools are likely to have a profound influence in the way we diagnose patients and plan our treatment 
approaches and mechanics. Below are a few examples of how big data can be leveraged to aid in decision making in orthodontics.

\subsection{Omics and orthodontics}

We are living in an exciting time of major technological advancements in genetics. The next-generation sequencing (NGS) technology has rapidly evolved since the time of human genome project in 2003 with a declining cost of US $\$ 10$ million in 2007 to $\$ 1500$ in $2015 .{ }^{19}$ NGS commonly refers to a technology of massively parallel sequencing of small fragments of DNA or RNA in a relatively short time. ${ }^{20}$ The whole-exome (WES) sequencing, whole-genome sequencing (WGS), epigenome and transcriptome are subset of NGS. The major steps involved in NGS process are the following: (a) DNA/RNA isolation from a cell or tissue type; (b) DNA/RNA library preparation; (c) sequencing of DNA/RNA library; and (d) variant or transcriptomic or epigenomic analyses using sophisticated computational tools. ${ }^{20}$ Before the advent of NGS, gene discovery approaches were rather a tedious and time-consuming process requiring large family pedigree to conduct linkage studies. This has changed dramatically with the reduced cost and improved NGS technology. In recent times, genes associated with several rare genetic disorders were discovered using NGS in a small cohort of paediatric population in a short amount of time. ${ }^{21}$

The NGS technology is largely underutilized in orthodontics. Traditionally, the orthodontists are interested in understanding the development and growth of the craniofacial complex with the hope to intervene and alter the abnormal growth patterns. Orthodontic researchers have always sought methods to predict growth in Class II or Class III patients. For such prediction, the orthodontists have relied upon phenotype-driven diagnostic methods such as cephalometric analyses (Steiner, Tweed, Ricketts, etc.). A few recent studies have identified the loci or genes for malocclusions. ${ }^{22,23}$ While the role of genetics and epigenetics in the development of craniofacial complex is not disputed, currently there is nothing in the orthodontist toolkit to employ genotype-driven diagnosis. To incorporate patient's genotype in the diagnostic process, genetic markers associated with the development of craniofacial complex need to be identified. Past studies that investigated for such markers had utilized either a candidate gene or gene panel approaches. What is rather needed is large-scale genomewide association studies (GWAS) using NGS. Associating such genetic markers through GWAS will help us provide genotype-driven diagnosis and render customized orthodontic care. Such information could be leveraged to aid in decision making in orthodontics.

Not all variations in the population could be explained by genetics alone. Epigenetics play an important and long-lasting role in early development, metabolism and health of an individual. ${ }^{24}$ The epigenetic changes commonly refer to cytosine methylation of DNA or post-translational modification of histone proteins. These epigenetic changes allow chromatin modifications to regulate gene expression. ${ }^{24}$ Such epigenetic changes have tremendous implication for understanding disease or population variation. These changes are largely influenced by external environmental factors such as diet, pollutants, temperature changes and stress. ${ }^{24,25}$ In the orthodontic literature, large emphasis has always been placed on the role of environment in the development of skeletal and dental malocclusions. With the recent technological advancements in NGS, the genomewide epigenetic marks could be easily identified in different cell and tissue types of the craniofacial complex. ${ }^{25}$ Future studies, using NGS, must focus on the identifying the role epigenetic changes in the normal and abnormal craniofacial development. Such information, in addition to conventional clinical and radiographic assessment, will greatly help clinician in rendering customized orthodontic care.

In addition to genetic and epigenetic changes, RNA modifications also contribute to regulating and fine-tuning gene expression. With the advent of RNA-seq, the investigation of transcriptomes and RNA modifications in health and disease are now feasible. ${ }^{26}$ Furthermore, mass spectrometry-based proteomics has tremendously advanced to perform quantitative analyses of proteomes; identify post-translational modifications; and study protein-protein interactions. ${ }^{27}$ These technologies have a very important role in orthodontics to enhance our care for our patients. For example, biological markers for orthodontic tooth movement (OTM) have been an area of great interest. Current studies on OTM are largely based on candidate gene approach comparing different groups of subjects treated with varying orthodontic force levels. The limited information derived from such studies has limited clinical application. If the studies on OTM were conducted using high-throughput transcriptome or proteomic analyses, then we will be able to identify different global players in the signalling pathway of OTM and identify the governing gene regulatory networks. These gene regulatory networks in OTM will pave way for targeted therapy.

Population genetics research has traditionally used convenient probabilistic model or its approximation with an assumption that the model describes the data sufficiently. These traditional models were validated using goodness-of-fit tests or examination of residuals. Interestingly, the amount of sequence data has increased 10-fold every year since 2002, contradicting the Moore's law that states computing power and storage capacity will double every 18 months. ${ }^{28}$ With the explosion of genomic data, the population genetic research is moving from "data modelling culture" to "algorithmic modelling culture" using machine-learning tools to identify relevant and meaningful information from the large data set. ${ }^{29}$ Furthermore, high-throughput methods such as GWAS, transcriptome, proteome and epigenome provide large volumes of data in a unidimensional view. ${ }^{30}$ Integrating these data will provide multidimensional view and help us understand the aetiopathogenesis of human disease. ${ }^{30}$ To analyse and integrate large volumes of such data require advanced and novel computational tools such as parallel computing and machine-learning techniques. Such techniques will allow us to identify patterns from structured and unstructured data. ${ }^{28}$ For example, machine-learning tools such as SPARCLE (sparse recovery of linear combinations of expression) and PEER have made great strides in identifying regulatory genes and associated pathways. ${ }^{28}$ This information has a potential for greater impact on translational 
biomedicine. Furthermore, novel machine-learning approaches are already available to study epigenetics and metabolic pathways. ${ }^{31,32}$ The advancements in NGS and the power of machine learning are underutilized in orthodontics. Future studies employing these technologies will create a paradigm shift in orthodontic diagnosis. The availability of omics data will lend an entirely new dimension to the delivery of orthodontic care. Some of the big data tools are likely to have a profound influence in the way we diagnose patients and plan our treatment approaches and mechanics.

\subsection{Imaging and orthodontics}

Imaging in orthodontics is an essential and integral part of assessment and treatment planning. Initial diagnosis and treatment follow-up have been traditionally evaluated predominantly on panoramic and cephalometric radiographs with supplemental use of intraoral radiographs as needed. However, this trend has witnessed a change since 1999 when cone-beam computed tomography $(C B C T)$ was introduced in dentistry. While the use of $C B C T$ in routine orthodontics has increased in the past decade, there is limited and mostly anecdotal evidence showing the importance of $\mathrm{CBCT}$ in routine orthodontic treatment. ${ }^{33}$ The CBCTs have been useful especially for patients with complex conditions in the oral and maxillofacial regions. СBCT enables a better understanding of the patient's anatomy, and data from these images can be integrated with photographs and 3D surface models to enable dynamic patient-specific anatomical reconstructions and the possibility of $3 \mathrm{D}$ treatment planning. ${ }^{33}$ With the vast array of structures that are visualized on these images, there is the possibility to develop various tools using algorithms to convert the raw data from these images into large data sets and to potentially use artificial intelligence to detect the presence of anatomical variations and/or diseases. ${ }^{34}$ There are a large number of emerging studies that are focused on mining the anatomical structure data based on predefined imaging features such as signal-tonoise ratio, windowing and levelling to enable the use of artificial intelligence to help detect subtle variations in anatomy and any incipient lesions which might not have been picked by humans. The technology is currently not fully mature to use in the craniofacial region and has limited applicability often focusing on a few conditions. With the complexity of structures in the craniofacial region and high prevalence of incidental findings on CBCT images, there is a need to have the images evaluated by an individual with advanced training in radiologic interpretation of the oral and maxillofacial complex to detect the presence of abnormal conditions and/or anatomical variations and artificial intelligence can be an excellent tool in facilitating this. ${ }^{35}$

\section{3 | Centralized data repositories}

The long-standing bane of orthodontics research has been the fact that a vast majority of clinical studies draw their samples from a single clinic (usually with a single provider), from a few clinics (with a few providers) or from university-based settings (both residents and attendings serve as primary providers in varying capacity). Consequently, the results and conclusions cannot be generalized, are not externally valid and may be a reflection of clustering of outcomes within clinic settings and/or providers. In terms of pooling high volumes of patient data to inform clinical decision making, dentistry is far behind structurally when compared to medical specialties. Moreover, dental medicine and orthodontics have little recorded large-scale outcomes data to fuel a knowledge base. Most of the big data studies in dental medicine and orthodontics have used hospital outcomes data because dental practices have been poor at recording outcomes and pooling patient data. There is a need for real-time, collaborative data pooling for the purpose of quality improvement in orthodontics. As most of the practices and university/hospitalbased clinical settings have transitioned to using electronic health record systems, creating centralized data repositories is imminent. While at first glance, this may appear to be an unviable option considering there is no consensus within our community on how we keep records, we need not look any further than the existing nationwide hospital databases. Every hospital across the entire United States has electronic health records, and there is large variation in how they record data. However, there are uniform data elements pertaining to patient demographics, diagnoses, treatments rendered and outcomes realized. These uniform data elements have been successfully combined across all hospitals to create large data sets such as the Nationwide Inpatient Sample, the Nationwide Emergency Department Sample and the State Ambulatory data sets. ${ }^{36}$ The Agency for Healthcare Research and Quality has partnered with different states and commercial vendors to create these data sets and make them available to the research community. This has created a tremendous wealth of knowledge and aided in clinical decision making and health policy reforms. In the orthodontics field, a few residency programmes or practices can partner to create problem/ disease specific data sets to examine orthodontic-related outcomes. In fact, such efforts are already underway. An example is the Anterior Open Bite Study of the National Dental Practice Based Research Network (Dental PBRN). This is a nationwide study funded by the NIH/NIDCR through the Dental PBRN. This study has been successful in collecting data from 347 anterior open-bite patients drawn from 91 different practitioners in both private practices and university-based residency programmes. ${ }^{37}$ Yet another example of a smaller scale regional study which has been successful in integrating records from 3 different private practices and two university-based programmes is the pilot phase of the Class II outcomes study funded by the American Association of Orthodontists Foundation. Both of these studies looked at a specific patient population (anterior open bites and severe Class II Division I malocclusions) and associated outcomes. With the current availability of interinstitutional operable information technology platforms, the speed of computing processing, and network security measures, more disease/ 
condition-specific data repositories can be established to examine clinical orthodontic outcomes.

\section{4 | CONCLUSIONS}

This article provides an overview of the current landscape of big data analytics in the healthcare sector and the common analytical tools employed to analyse large data sets. There are several avenues for the field of orthodontics to make use of big data analytics to improve clinical outcomes.

\section{ACKNOWLEDGEMENT}

The study was funded by the American Association of Orthodontists Foundation Biomedical Research Awards to Dr. Veeasathpurush Allareddy in years 2016 and 2017.

\section{CONFLICTS OF INTEREST}

None of the authors of the manuscript have any conflicts of interest to report.

\section{ORCID}

Veerasathpurush Allareddy (iD http://orcid.org/0000-0001-8199-6206

\section{REFERENCES}

1. Hansen MM, Miron-Shatz T, Lau AY, Paton C. Big data in science and healthcare: a review of recent literature and perspectives. Contribution of the IMIA Social Media Working Group. Yearb Med Inform. 2014;9:21-26.

2. Mehta N, Pandit A. Concurrence of big data analytics and healthcare: a systematic review. Int J Med Inform. 2018;114:57-65.

3. NIH Strategic Plan for Data Science. www link https://datascience. nih.gov/sites/default/files/NIH_Strategic_Plan_for_Data_Science Final_508.pdf. Accessed October 10, 2018.

4. Ristevski B, Chen M. Big data analytics in medicine and healthcare. J Integr Bioinform. 2018;15(3):1-5.

5. Roski J, Bo-Linn GW, Andrews TA. Creating value in health care through big data: opportunities and policy implications. Health Aff (Millwood). 2014;33(7):1115-1122.

6. Rumsfeld JS, Joynt KE, Maddox TM. Big data analytics to improve cardiovascular care: promise and challenges. Nat Rev Cardiol. 2016;13(6):350-359.

7. Wu PY, Cheng CW, Kaddi CD, Venugopalan J, Hoffman R, Wang MD. Omic and electronic health record big data analytics for precision medicine. IEEE Trans Biomed Eng. 2017;64(2):263-273.

8. West JL, Fargen KM, Hsu W, Branch CL Jr, Couture DE. A review of Big Data analytics and potential for implementation in the delivery of global neurosurgery. Neurosurg Focus. 2018;45(4):E16.

9. Belle A, Thiagarajan R, Soroushmehr SM, Navidi F, Beard DA, Najarian K. Big data analytics in healthcare. Biomed Res Int 2015;2015:370194.

10. Johnson KW, Torres Soto J, Glicksberg BS, et al. Artificial intelligence in cardiology. J Am Coll Cardiol. 2018;71(23):2668-2679.
11. Raghupathi W, Raghupathi V. Big data analytics in healthcare: promise and potential. Health Inf Sci Syst. 2014;7(2):3.

12. Yang C, Li C, Wang Q, Chung D, Zhao H. Implications of pleiotropy: challenges and opportunities for mining big data in biomedicine. Front Genet. 2015;30(6):229.

13. Steyerberg EW, Eijkemans MJ, Van Houwelingen JC, Lee KL, Habbema JD. Prognostic models based on literature and individual patient data in logistic regression analysis. Stat Med. 2000;19(2):141-160.

14. Cobb AN, Benjamin AJ, Huang ES, Kuo PC. Big data: more than big data sets. Surgery. 2018;164(4):640-642.

15. Dinov ID. Methodological challenges and analytic opportunities for modeling and interpreting Big Healthcare Data. Gigascience. 2016;25(5):12

16. Handelman GS, Kok HK, Chandra RV, Razavi AH, Lee MJ, Asadi H. eDoctor: machine learning and the future of medicine. J Intern Med. 2018;284:603-619.

17. Pashazadeh A, Navimipour NJ. Big data handling mechanisms in the healthcare applications: a comprehensive and systematic literature review. J Biomed Inform. 2018;82:47-62.

18. Alonso-Betanzos A, Bolón-Canedo V. Big-data analysis, cluster analysis, and machine-learning approaches. Adv Exp Med Biol. 2018;1065:607-626.

19. Payne K, Gavan SP, Wright SJ, Thompson AJ. Cost-effectiveness analyses of genetic and genomic diagnostic tests. Nat Rev Genet. 2018;19(4):235-246.

20. Goodwin S, McPherson JD, McCombie WR. Coming of age: ten years of next-generation sequencing technologies. Nat Rev Genet. 2016;17(6):333-351.

21. Wright CF, FitzPatrick DR, Firth HV. Paediatric genomics: diagnosing rare disease in children. Nat Rev Genet. 2018;19(5):253-268.

22. Cruz CV, Mattos CT, Maia JC, et al. Genetic polymorphisms underlying the skeletal Class III phenotype. Am J Prthod Dentofacial Orthop. 2017;151:700-707.

23. da Fontoura CSG, Miller SF, Wehby GL, etal. Candidate gene analyses of skeletal variation malocclusion. J Dent Res. 2015;94(7):913-920.

24. Feil R, Fraga MF. Epigenetics and the environment: emerging patterns and implications. Nat Rev Genet. 2012;13(2):97-109.

25. Taudt A, Colomé-Tatché M, Johannes F. Genetic sources of population epigenomic variation. Nat Rev Genet. 2016;17(6):319-332.

26. Helm M, Motorin Y. Detecting RNA modifications in the epitranscriptome: predict and validate. Nat Rev Genet. 2017;18(5):275-291.

27. Altelaar AF, Munoz J, Heck AJ. Next-generation proteomics: towards an integrative view of proteome dynamics. Nat Rev Genet. 2013;14(1):35-48

28. Berger B, Peng J, Singh M. Computational solutions for omics data. Nat Rev Genet. 2013;14(5):333-346.

29. Schrider DR, Kern AD. Supervised machine learning for population genetics: a new paradigm. Trends Genet. 2018;34(4):301-312.

30. Hawkins RD, Hon GC, Ren B. Next-generation genomics: an integrative approach. Nat Rev Genet. 2010;11(7):476-486.

31. Holder LB, Haque MM, Skinner MK. Machine learning for epigenetics and future medical applications. Epigenetics. 2017;12(7):505-514.

32. Cuperlovic-Culf M. Machine learning methods for analysis of metabolic data and metabolic pathway modeling. Metabolites. 2018;8(1):E4.

33. American Academy of Oral and Maxillofacial Radiology. Clinical recommendations regarding use of cone beam computed tomography in orthodontics. Position statement by the American Academy of Oral and Maxillofacial Radiology.Oral Surg Oral Med Oral Pathol Oral Radiol 2013;116(2):238-257.

34. Hosny A, Parmar C, Quackenbush J, Schwartz LH, Aerts HJW. Artificial intelligence in radiology. Nat Rev Cancer 2018;18(8):500-510. 
35. Allareddy V, Vincent SD, Hellstein JW, Qian F, Smoker WR, Ruprecht A. Incidental findings on cone beam computed tomography images. Int J Dent. 2012;2012:871532.

36. HCUP Databases. Healthcare Cost and Utilization Project (HCUP). August 2018. Agency for Healthcare Research and Quality, Rockville, MD. www link is www.hcup-us.ahrq.gov/databases.jsp. Accessed October 10, 2018.

37. Choi KW, Ko HC, Todoki LS, et al. The National Dental Practice-Based Research Network adult anterior open bite study: a description of the practitioners and patients. Angle Orthod 2018;88:675-683.
How to cite this article: Allareddy $\mathrm{V}$, Rengasamy Venugopalan S, Nalliah RP, Caplin JL, Lee MK, Allareddy V. Orthodontics in the era of big data analytics. Orthod Craniofac Res.

2019;22(Suppl. 1):8-13. https://doi.org/10.1111/ocr.12279 